Situação: O preprint foi submetido para publicação em um periódico

\title{
Colapso na Saúde em Manaus: o fardo de não aderir às medidas não farmacológicas de redução da transmissão da COVID-19
}

Ivana Cristina de Holanda Cunha Barreto, Valter Costa, Ronaldo Fernandes Ramos, Luciana Gonzaga de Oliveira, Natália Regina Alves Vaz Martins, Fabrício Vieira Cavalcante, Luiz Odorico Monteiro de Andrade, Leonor Maria Pacheco Santos

https://doi.org/10.1590/SciELOPreprints.1862

Este preprint foi submetido sob as seguintes condições:

- O autor submissor declara que todos os autores responsáveis pela elaboração do manuscrito concordam com este depósito.

- Os autores declaram que estão cientes que são os únicos responsáveis pelo conteúdo do preprint e que o depósito no SciELO Preprints não significa nenhum compromisso de parte do SciELO, exceto sua preservação e disseminação.

- Os autores declaram que a pesquisa que deu origem ao manuscrito seguiu as boas práticas éticas e que as necessárias aprovações de comitês de ética de pesquisa estão descritas no manuscrito, quando aplicável.

- Os autores declaram que os necessários Termos de Consentimento Livre e Esclarecido de participantes ou pacientes na pesquisa foram obtidos e estão descritos no manuscrito, quando aplicável.

- Os autores declaram que a elaboração do manuscrito seguiu as normas éticas de comunicação científica.

- Os autores declaram que o manuscrito não foi depositado e/ou disponibilizado previamente em outro servidor de preprints ou publicado em um periódico.

- O autor submissor declara que as contribuições de todos os autores estão incluídas no manuscrito.

- O manuscrito depositado está no formato PDF.

- Os autores declaram que caso o manuscrito venha a ser postado no servidor SciELO Preprints, o mesmo estará disponível sob licença Creative Commons CC-BY.

- Caso o manuscrito esteja em processo de avaliação ou sendo preparado para publicação mas ainda não publicado por um periódico, os autores declaram que receberam autorização do periódico para realizar este depósito.

Submetido em (AAAA-MM-DD): 2021-02-13

Postado em (AAAA-MM-DD): 2021-02-18 


\section{Colapso na Saúde em Manaus: o fardo de não aderir às medidas não farmacológicas de redução da transmissão da COVID-19}

\section{Health Collapse in Manaus: the burden of not adhering to non-pharmacological measures to reduce the transmission of COVID-19}

Ivana Cristina de Holanda Cunha Barreto

ivana.barreto24@gmail.com

(ORCID 0000-0001-8447-3654)

Fundação Oswaldo Cruz: Eusébio, Ceará, BR

Raimundo Valter Costa Filho

valter.costa@ifce.edu.br

(ORCID 0000-0002-2456-8819)

Instituto Federal do Ceará: Fortaleza, CE, BR

Universidade Federal Rural do Semi-Árido: Mossoró, RN, BR

Ronaldo Fernandes Ramos

ronaldo@ifce.edu.br

(ORCID 0000-0003-2841-1787)

Instituto Federal do Ceará: Fortaleza, CE, BR

Luciana Gonzaga de Oliveira.

lucianagoliveira7@gmail.com.

(ORCID 0000-0003-2399-3052)

Escola de Governo Fiocruz Brasília, Distrito Federal.

Natália Regina Alves Vaz Martins.

natalia.ravm@gmail.com.

(ORCID 0000-0003-1162-8795)

Programa de Pós-Graduação em Saúde Coletiva, Faculdade de Ciências da Saúde, Universidade de Brasília, Brasil.

Fabrício Vieira Cavalcante.

fabricioocavalcante@gmail.com.

(ORCID 0000-0002-8706-0457)

Programa de Pós-Graduação em Saúde Coletiva, Faculdade de Ciências da Saúde, Universidade de Brasília, Brasil.

Luiz Odorico Monteiro de Andrade.

Odorico.monteiro@fiocruz.br

(ORCID (0000-0002-3335-0619)

Pesquisador Especialista em Ciência, Tecnologia, Produção e Inovação da FIOCRUZ Ceará.

Faculdade de Medicina de Sobral - Universidade Federal do Ceará

Leonor Maria Pacheco Santos

leopac@unb.br

(ORCID 0000-0002-6739-6260),

Departamento de Saúde Coletiva, Faculdade de Ciências da Saúde

Universidade de Brasília, Brasil.

Financiamento: Chamada Pública MCTI/CNPq/CT-Saúde/MS/SCTIE/Decit No 07/2020 


\section{RESUMO}

O objetivo desse artigo é comparar o comportamento da COVID-19 em Manaus e Fortaleza, dois epicentros da pandemia em 2020, analisando medidas legais dos governos locais e níveis de isolamento social. Definiu-se um algoritmo para calcular o Índice de Permanência Domiciliar (IPD), com dados do Google Mobility Report. Analisou-se a linha do tempo dos Decretos, a evolução do IPD, da incidência de COVID-19 e do número de óbitos de março/2020 a janeiro/2021. A população de Fortaleza esteve exposta a medidas de distanciamento social mais consistentes que as de Manaus. Maior permanência domiciliar foi observada de março a maio de 2020 e Fortaleza atingiu níveis mais elevados e duradouros. A partir de junho o IPD caiu, sobretudo em Manaus, atingindo níveis abaixo de zero no final de dezembro. Como agravante, o governo decreta amplo isolamento em Manaus em 23/12/2020, mas após protestos, revoga-o em 26/12/2020. Uma Decisão Judicial determina o fechamento completo em Manaus em 02/01/2021, mas foi tarde demais: o SUS entra em colapso com aumento exponencial dos óbitos. Em Fortaleza a demanda aos serviços de saúde está elevada, mas sob controle. Considera-se que somente a aplicação rigorosa de medidas não farmacológicas e imunização em massa poderão evitar mais mortes.

Palavras-Chave: Infecções por Coronavírus, pandemia, decretos, isolamento social, Sistema Único de Saúde.

\section{ABSTRACT}

The purpose of this article is to compare the evolution of COVID-19 in Manaus and Fortaleza, two epicenters of the pandemic in 2020, analyzing legal measures by local governments and levels of social isolation. An algorithm was defined to calculate the Homestay Index (HSI), using data from the Google Mobility Report. The Decree's timeline, the HSI evolution, the incidence of COVID-19 and the number of deaths from March/2020 to January/2021 were analyzed. The population of Fortaleza was exposed to more consistent measures of social distance, than those of Manaus. Longer homestay was observed from March to May 2020 and Fortaleza achieved higher and more lasting levels. As of June 2020, the HSI fell, notably in Manaus, reaching levels below zero in late December. As an aggravating factor, the government decreed ample isolation in Manaus on December 23/2020, but after protests, it repeals it on December 26/2020. A Judicial Decision determines the complete closure in Manaus on January 02/2021, but it was too late: SUS collapses with an exponential increase in deaths. In Fortaleza, the demand for health services is high, but under control. We consider that only the strict application of non-pharmacological measures and mass immunization can prevent further deaths. KEYWORDS: COVID-19, decrees, social isolation, Unified Health System, pandemic. 


\section{INTRODUÇÃO}

O primeiro caso da COVID-19 no Brasil foi registrado em 26 de fevereiro de 2020 e a transmissão comunitária foi reconhecida em todo o território em 20 de março desse mesmo ano ${ }^{1}$. Em virtude da inexistência de uma vacina e de tratamentos medicamentosos específicos e eficazes, a Organização Mundial da Saúde (OMS), baseando-se na experiência de controle de neo epidemias virais, como a Middle East Respiratory Syndrome (MERS), recomendou medidas não farmacológicas para o controle da pandemia de COVID-19². Entre as medidas não farmacológicas está o distanciamento social, desencadeado por meio da paralisação ou redução de atividades não essenciais nos setores públicos e privados, como atividades da administração pública, atividades escolares, comerciais, industriais, de turismo, eventos em geral, entre outras. A associação de medidas de distanciamento social às medidas de controle sanitário, como a investigação de casos suspeitos de COVID-19 e seus contatos, a imposição de quarentena aos casos suspeitos e confirmados de COVID-19, a testagem de todos os casos suspeitos e de grupos mais expostos ou vulneráveis, e, ademais, a recomendação do uso de máscaras, álcool gel e sabão para higiene das mãos, são outras medidas recomendadas pela OMS. Medidas sociais como a garantia de renda mínima e alimentação para populações vulneráveis e sem emprego, são também fundamentais para o cumprimento do distanciamento social. A implementação dessas medidas exige uma coordenação nacional interinstitucional e intersetorial, com clara centralidade política voltada ao controle da pandemia, baseada em evidências científicas e alinhada à $\mathrm{OMS}^{\mathbf{3}, \mathbf{4}}$.

Segundo dados do Ministério da Saúde, de fevereiro de 2020 até 31 de janeiro de 2021, ocorreram no Brasil 9.202.791 casos confirmados de COVID-19 e 224.534 óbitos pela doença, com uma taxa de letalidade de 2,5\% $\%^{5}$. Além de continental, o Brasil é um país federativo com três esferas de governo, sendo o único do mundo com mais de 100 milhões de habitantes com um sistema de saúde público, constitucionalmente universal, e descentralizado em nível dos municípios ${ }^{6}$. Porém, toda a potência do Sistema Único de Saúde (SUS) tem sido minada por medidas econômicas de austeridade fiscal, como a Emenda Constitucional 95. Ademais, vem ocorrendo o enfraquecimento de suas instâncias interfederativas de gestão consensual do SUS, em especial a Comissão Intergestores Tripartite, composta por representações das gestões federal, estadual e municipal. A quebra das decisões consensuais entre os gestores ficou claras pela recente manifestação do Conselho Nacional de Secretários de Saúde (CONASS), se declarando contrário à permissão dada pelo governo federal para compra de 33 milhões de doses de vacinas por empresas privadas, uma vez que esta compra pode enfraquecer o Programa 
Nacional de Imunizações, e, consequentemente, prejudicar o controle da pandemia de COVID-197.

Manaus, capital do Amazonas, já havia sido palco de elevadas taxas de incidência e mortalidade pela COVID-19 em maio de 2020, o que causou inclusive colapso do sistema funerário durante a primeira onda da pandemia, trazendo sofrimento à sua população. Nas últimas semanas de dezembro de 2020 e primeiras semanas de janeiro de 2021, nova onda de casos deixa a cidade em choque, agora trazendo o colapso do sistema municipal de saúde por falta de leitos de enfermaria, leitos de UTI e oxigênio. Enquanto isto, outras capitais do país conseguem manter o controle, ao menos até o momento, da demanda aos serviços de saúde. É o caso de Fortaleza, que apresentou um pico acentuado de óbitos de COVID-19 logo após Manaus, em junho de 2020. No presente artigo os autores pretendem analisar se as medidas legais dos governos locais e os níveis de isolamento social, podem ter contribuído para o colapso do SUS no município de Manaus, comparando com o comportamento da pandemia de COVID-19 em Fortaleza, dois municípios que em meados de 2020 foram considerados como epicentros da primeira onda da pandemia.

\section{METODOLOGIA}

As capitais em estudo foram comparadas quanto a alguns indicadores sócio demográficos e de saúde, como IDHM e PIB per capita ${ }^{\mathbf{8}, \mathbf{9}}$, população estimada ${ }^{\mathbf{1 0}}$, densidade demográfica urbana ${ }^{\mathbf{9}}$ e taxa de escolarização de 6 a 14 anos ${ }^{9}$. A evolução entre março e junho de 2020 da taxa de médicos foi obtida pela razão entre o número de médicos disponíveis ${ }^{\mathbf{1 1}}$ e a população estimada para $2020^{\mathbf{1 0}}$, multiplicada por 1000 habitantes. De modo similar as taxas de leitos de UTI foram calculadas como razão entre número de leitos de UTI adultos disponíveis no Cadastro Nacional de Estabelecimento de Saúde (CNES) no período identificado ${ }^{11}$ e a estimativa de população de adultos em cada capital com base no censo/2010 ${ }^{\mathbf{1 2}}$, multiplicada por 1000 adultos.

Para inferir a redução da circulação de pessoas e, portanto, diminuição da probabilidade de transmissão do vírus, definiu-se o Índice de Permanência Domiciliar (IPD), calculado com base nos dados publicados no Google Mobility Report ${ }^{\mathbf{1 3}}$. Este é um relatório contendo a variação percentual do tempo de permanência médio dos indivíduos em locais residenciais, local de Trabalho, Mercado\&Farmácia, Parques, Trânsito\&Estações em comparação com a moda de permanência média para cada dia da semana no período pré-pandemia (03/01/2020 a 06/02/2020). O IPD é um índice relativo e visa comparar a efetividade das medidas de distanciamento social coordenadas pelo poder público entre localidades. Quanto maior o índice, 
maior a permanência residencial e menor a circulação de pessoas em áreas públicas, sugerindo uma diminuição da probabilidade de exposição de pessoas suscetíveis ao novo coronavírus.

Para construir o algoritmo e calcular o Índice de Permanência Domiciliar (IPD), a partir dos dados do Google Mobility Report, toma-se:

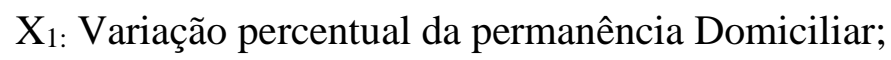

$\mathrm{X}_{2}$ : Variação percentual da permanência em Mercados\&Farmácias;

$\mathrm{X}_{3}$ : Variação percentual da permanência no Trabalho;

$\mathrm{X}_{4}$ : Variação percentual da permanência no Trânsito\&Estações;

$\mathrm{X}_{5}$ : Variação percentual da permanência no Varejo\&Lazer;

$\mathrm{X}_{6}$ : Variação percentual da permanência em Parques;

O somatório das diferenças relativas à permanência domiciliar $\left(\mathrm{D}_{\mathrm{D}}\right)$ é, então:

$$
D_{D}=\left(X_{1}-X_{2}\right)+\left(X_{1}-X_{3}\right)+\left(X_{1}-X_{4}\right)+\left(X_{1}-X_{5}\right)+\left(X_{1}-X_{6}\right)
$$

A variação média da diferença entre taxa domiciliar e as outras resulta no IPD:

$$
\begin{gathered}
I P D=\frac{D_{D}}{5}=\frac{\left(X_{1}-X_{2}\right)+\left(X_{1}-X_{3}\right)+\left(X_{1}-X_{4}\right)+\left(X_{1}-X_{5}\right)+\left(X_{1}-X_{6}\right)}{5} \\
I P D=\frac{5 X_{1}-\left(X_{2}+X_{3}+X_{4}+X_{5}+X_{6}\right)}{5} \\
I P D=X_{1}-\frac{\left(X_{2}+X_{3}+X_{4}+X_{5}+X_{6}\right)}{5}
\end{gathered}
$$

Ou:

IPD = Domiciliar $-\underline{\text { Mercados\&Farmácias + Trabalho +Trânsito\&Estações + Varejo\&Lazer + Parques }}$ 5

O IPD é, então, a média das diferenças entre a permanência residencial e nos outros locais. Vale ressaltar que, apesar de ser uma média entre percentuais, não representa o percentual de permanência domiciliar e sim um índice onde a pontuação positiva significa menor circulação de pessoas em relação ao período pré-pandemia (03/01/2020 à 06/02/2020).

Os dados sobre número de casos notificados e óbitos foram coletados do portal Coronavírus Brasil $^{5}$. A taxa de incidência foi calculada considerando a população estimada disponível no Sistema IBGE de Recuperação Automática (SIDRA) ${ }^{\mathbf{1 4}}$.

A recuperação e extração de dados sobre Decretos e Leis que instituíram as medidas não farmacológicas para contenção da epidemia pelos governos estaduais, datas da adoção de 
isolamento social e de relaxamento aplicadas aos estados do Amazonas e Ceará no período de fevereiro de 2020 a 31 de janeiro de 2021 , foram extraídos em sítio eletrônico ${ }^{\mathbf{1 5}}$.

\section{RESULTADOS}

$\underline{\text { Comparação de indicadores selecionados em Fortaleza e Manaus }}$

A comparação de indicadores sócio demográficos e de saúde selecionados nas duas capitais estudadas é apresentada no Quadro 1. Quanto aos indicadores IDHM, população estimada e taxa de escolarização não há grandes disparidades. A densidade demográfica urbana em Fortaleza é o dobro da observada em Manaus, diferença que poderia ter reflexos importantes sobre a taxa de transmissão da COVID-19. Manaus também se compara positivamente em relação ao PIB per capita, que é $43 \%$ mais elevado que o de Fortaleza. Por outro lado, todos os indicadores de assistência à saúde em Fortaleza são mais favoráveis, com maior disponibilidade de leitos de enfermaria e de UTI, bem como maior razão de médicos por habitante.

Quadro 1. Indicadores sociodemográficos e de saúde selecionados. Fortaleza e Manaus, 2010 e 2020 .

\begin{tabular}{|c|c|c|c|}
\hline Indicador & Data-base & Manaus & Fortaleza \\
\hline $\mathrm{IDHM}^{8}$ & 2010 & 0,737 & 0,754 \\
\hline População estimada ${ }^{\mathbf{1 0}}$ & 2020 & 2.219 .580 & 2.686 .612 \\
\hline População em 2010 (Censo) $^{\mathbf{1 2}}$ & 2010 & 1.802 .014 & 2.452 .185 \\
\hline Área total ${ }^{9}$ & 2010 & $11.491,09 \mathrm{~km}^{2}$ & $314,93 \mathrm{~km}^{2}$ \\
\hline Área urbana ${ }^{12}$ & 2010 & $859,86 \mathrm{~km}^{2}$ & $314,93 \mathrm{~km}^{2}$ \\
\hline Densidade demográfica urbana $^{12}$ & 2010 & $2.095 \mathrm{hab} / \mathrm{km}^{2}$ & $7.786 \mathrm{hab} / \mathrm{km}^{2}$ \\
\hline PIB per capita 9 & 2018 & $\mathrm{R} \$ 36.445,75$ & $\mathrm{R} \$ 25.356,73$ \\
\hline Taxa de escolarização 6 a 14 anos 9 & 2010 & $94,2 \%$ & $96,1 \%$ \\
\hline \multirow{2}{*}{$\begin{array}{l}\text { Razão de médicos por } 1.000 \\
\text { habitantes }\end{array}$} & Mar 2020 & 1,66 & 2,55 \\
\hline & Jun 2020 & 1,68 & 2,63 \\
\hline \multirow{2}{*}{ Taxa de leitos UTI por adulto ${ }^{11}$} & Mar 2020 & 0,22 & 0,53 \\
\hline & Jun 2020 & 0,38 & 0,55 \\
\hline
\end{tabular}


Período de março a novembro de 2020: a primeira onda da pandemia

As primeiras medidas de contingência não farmacológicas, como uso de máscaras, isolamento social e restrição ao funcionamento de estabelecimentos, foram editadas no mesmo dia nos dois epicentros da COVID-19 analisados. A Figura 1 mostra a linha do tempo das medidas de isolamento e de relaxamento em Fortaleza e Manaus, determinadas por Decretos estaduais, Leis e Decisão Judicial, publicados de 16 de março a 31 de janeiro de 2021, comparada ao Índice de Permanência Domiciliar (IPD), cotejada com os dados epidemiológicos de taxas de incidência e média móvel do número de óbitos registrados no período.

O estado do Ceará publicou um total de nove Decretos (seis até 30/11/2020 e três entre 01/12/2020 e 31/01/2021). Foram quatro medidas legais de isolamento publicadas entre 16 e 23 de março, que foram prorrogados de 15 em 15 dias, até o dia 11 de julho, quando houve uma programação de reabertura para cada tipo de estabelecimento, desde que respeitando a quantidade de pessoas em relação à capacidade do local, condições de higiene, distâncias entre pessoas e tempo de espera.

Já o estado do Amazonas editou um total de 14 Decretos (oito até 30/11/2020 e seis entre 1/12/2020 e 31/01/2021). Os cinco Decretos de isolamento publicados logo no início, entre 16 e 31 de março, foram prorrogados ao longo dos meses, de 15 em 15 dias, até $1^{\circ}$ de junho. $\mathrm{O}$ primeiro Decreto de relaxamento reabriu Shoppings; logo a seguir bares, restaurantes, reuniões presenciais e depois lojas de brinquedo, papelaria e escritórios. No entanto, os decretos de abertura impunham condições para cada estabelecimento como medidas de higiene, menor quantidade de pessoas em relação à capacidade do local, distâncias entre pessoas e tempo de espera para atendimento. 

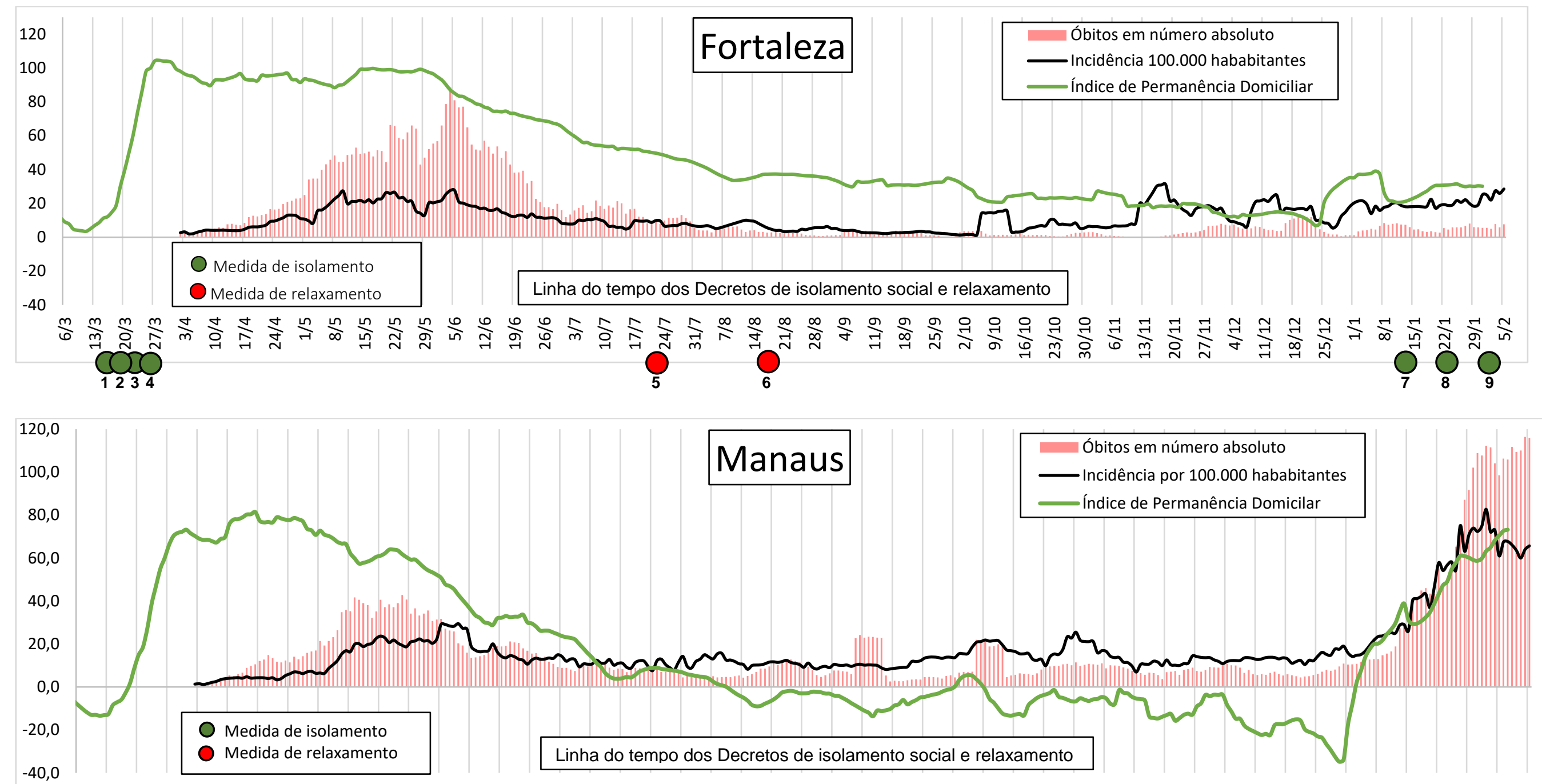

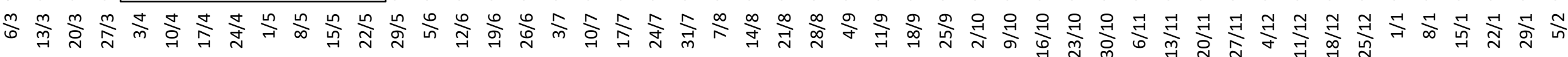
$\mathrm{OOO}_{101121314}$ $\bigcirc$ $\bigcirc_{16}$ 
FORTALEZA - Decretos estaduais de contenção e de relaxamento

1. Decreto № 33510 de 16 de março de 2020: suspende reuniões e eventos municipais, viagens oficiais e isola pessoas com diagnóstico de COVID

2. Decreto № 33519 de 19 de março de 2020: suspende aulas (públicas/particulares), estabelecimentos comerciais não essenciais, eventos mais 100 pessoas, atendimento presencial pelos órgãos públicos. 3. Decreto № 33521 de 21 de março de 2020: recomenda que os atendimentos sejam realizados por drive-tru ou entrega

4. Decreto №33523 de 23 de março de 2020: estabelece como locais essenciais padarias, supermercados e oficinas de carro. (Prazos de decretos de isolamento prorrogados de 15 em 15 dias até 11 de julho) 5. Decreto № 33671 de 11 de julho de 2020: regionalização do isolamento e programação de reabertura para que cada tipo de estabelecimento.

6. Decreto № 33704 de 05 de agosto de 2020: impõe condições para cada estabelecimento (número de pessoas e distância em relação à capacidade do local, condições de higiene, e tempo de espera)

7. Decreto № 33899, de 09 de janeiro de 2021: ficam prorrogadas, no Estado do Ceará, as medidas de isolamento social até o dia 31 de janeiro 2021.

8. Decreto № 33904, de 21 de janeiro de 2021, até 31/01/2021 permanece em vigor, no Estado do Ceará, as medidas de isolamento social previstas no Decreto ํo 33.519 , de 19 de março de 2020 .

9. Decreto № 33913, de 30 de janeiro de 2021: até 7 de fevereiro de 2021, permanece em vigor, no Estado do Ceará, as medidas de isolamento social previstas no Decreto no 33.519 , de 19 de março 2020

MANAUS - Decretos estaduais de contenção e de relaxamento

10. Decreto № 42061 de 16 de março de 2020: suspende aulas públicas e particulares, reuniões e eventos municipais, viagens oficiais e isola pessoas com diagnóstico de COVID-19.

11. Decreto № 42063 de 17 de março de 2020: suspende estabelecimentos comerciais não essenciais, eventos + 100 pessoas (shows).

12. Decreto № 42085 de 18 de março de 2020: suspende atendimento presencial ao público pelos órgãos públicos.

13. Decreto № 42087 de 19 de março de 2020 : suspende transporte rodoviário intermunicipal

14. Decreto № 42145 de 31 de março de 2020 : fechamento das visitas nas penitenciárias (Os prazos dos decretos de isolamento foram prorrogados de 15 em 15 dias, até 28 de maio.)

15. Decreto № 42330 de 28 de maio de 2020: (1) abertura de shoppings, órgãos públicos, templos religiosos dia 01/06; (2) bares e restaurantes dia 06/06; (3) reuniões presenciais dia 07/06; (4) lojas brinquedo, papelaria e escritório dia 15/06. Impõe condições para cada estabelecimento: quantidade de pessoas relativo à capacidade local, condições de higiene, distância entre pessoas e tempo de espera. 16. Decreto № 4906, de 18 de setembro de 2020: suspende por 60 (sessenta) dias, o acesso à praia do Complexo Turístico da Ponta Negra.

17. Lei № 2.709, de 27 de novembro de 2020: reabertura de restaurantes, padarias, panificadoras, supermercados e estabelecimentos que forneçam refeição no município de Manaus, com medidas restritivas 18. Decreto № 43234, de 23 de dezembro de 2020: proíbe, no período de 28/12/2020 a 11/01/2021, a abertura do comércio não essencial e a realização de reuniões comemorativas, inclusive de Ano Novo. 19. Decreto № 43236, de 28 de dezembro de 2020: revoga o fechamento do comércio não essencial anteriormente determinado pelo Decreto № 43434 .

20. Decisão judicial de 02 de janeiro de 2021, por solicitação da Defensoria Pública do Amazonas, mantém a validade do Decreto № 43234, proibindo a abertura do comércio não essencial.

21. Decreto № 5001, de 04 de janeiro de 2021: declara situação emergencial no Município de Manaus, pelo período de 180 (cento e oitenta dias), em razão da pandemia causada pelo novo Coronavírus. 22. Decreto № 4999, de 04 de janeiro de 2021: suspensos, até 31/03/2021, no âmbito da Administração Pública Municipal, todo e qualquer atendimento ao público de forma presencial.

23. Decreto № 43303, de 23 de janeiro de 2021: amplia a restrição temporária de circulação de pessoas em vias públicas, em todos os municípios do AM, durante as 24 h do dia (LOCKDOWN).

Figura 1 - Médias Móveis para os últimos 7 dias da taxa de incidência de COVID-19 por 100.000 habitantes, número absoluto de óbitos, Índice de Permanência Domiciliar e linha do tempo das medidas legais de isolamento social e relaxamento. Fortaleza e Manaus 2020-2021. 
Nota-se que em março, logo após a publicação das primeiras medidas de contenção, o IPD mostrou crescimento expressivo em ambas as capitais. No entanto, em Manaus, o índice foi bem menor e se manteve por um período mais curto, passando a apresentar valores negativos a partir de agosto, indicando que a população permanecia mais tempo na rua do que em casa. No estado do Amazonas as aberturas foram autorizadas entre $1^{\circ}$ e 15 de junho, ainda em plena epidemia em Manaus, tanto do ponto de vista da incidência, quanto dos óbitos por COVID-19. O primeiro Decreto de abertura, datado de 01/6/2020, priorizou o funcionamento de shoppings, órgãos públicos e templos religiosos. Já em Fortaleza, a permanência domiciliar foi bem maior e decaiu lentamente, mesmo após os Decretos de abertura em julho e agosto, permanecendo em valores mais baixos, mas ainda positivos, até o final de novembro de 2020. Em Fortaleza, as aberturas foram autorizadas a partir de 11 de julho, quando já havia tendência de queda tanto na incidência de casos como nos óbitos por COVID-19 (Figura 1).

Ao longo deste primeiro período de estudo, a análise mais detalhada por local de permanência IPD apresenta valores negativos em Manaus a partir de agosto, em decorrência de maior permanência em Trânsito\&Estações e Trabalho, sobretudo entre outubro e novembro. Por outro lado, em Fortaleza o padrão de permanência da população é distinto, com predomínio de Mercado\&Farmácia, seguido por Trânsito\&Estações (Figura 2).

\section{$\underline{\text { Período de dezembro de } 2020 \text { a janeiro 2021: o colapso em Manaus }}$}

No dia 23 de dezembro, o governador do Amazonas publicou o Decreto 43.234 determinando que shoppings, flutuantes, bares e estabelecimentos do comércio não essenciais não poderiam funcionar pelo período de 15 dias. Proibia, também, reuniões comemorativas em espaços públicos, clubes e condomínios, inclusive na noite de réveillon. Já academias, mercados, feiras, cartórios e oficinas mecânicas teriam o funcionamento permitido (Figura 1).

A população, empresários, funcionários de lojas, camelôs, protestam de maneira enfática contra as medidas restritivas que determinavam o fechamento do comércio. Atendendo à pressão popular, o governador recuou por meio do Decreto 43.236 e liberou o funcionamento do comércio geral, com apenas algumas restrições de horário (Figura 1).

Neste período o IPD apresenta valores negativos em Manaus, em decorrência de maior permanência em Varejo\&Lazer, Trânsito\&Estações, Mercados\&Farmácias (Figura 2), indicando movimentação típica de fim de ano já a partir do início de outubro, acentuando-se até o final de dezembro. 


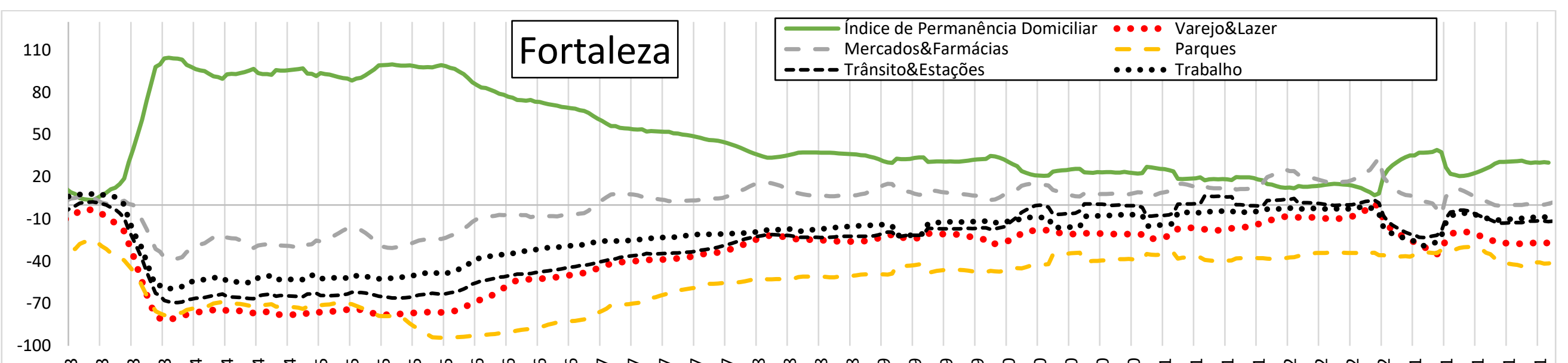

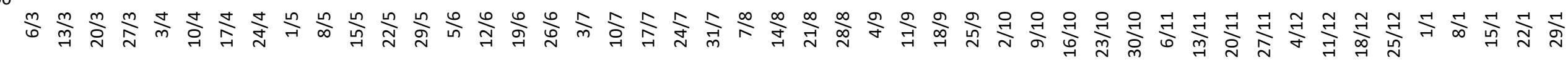

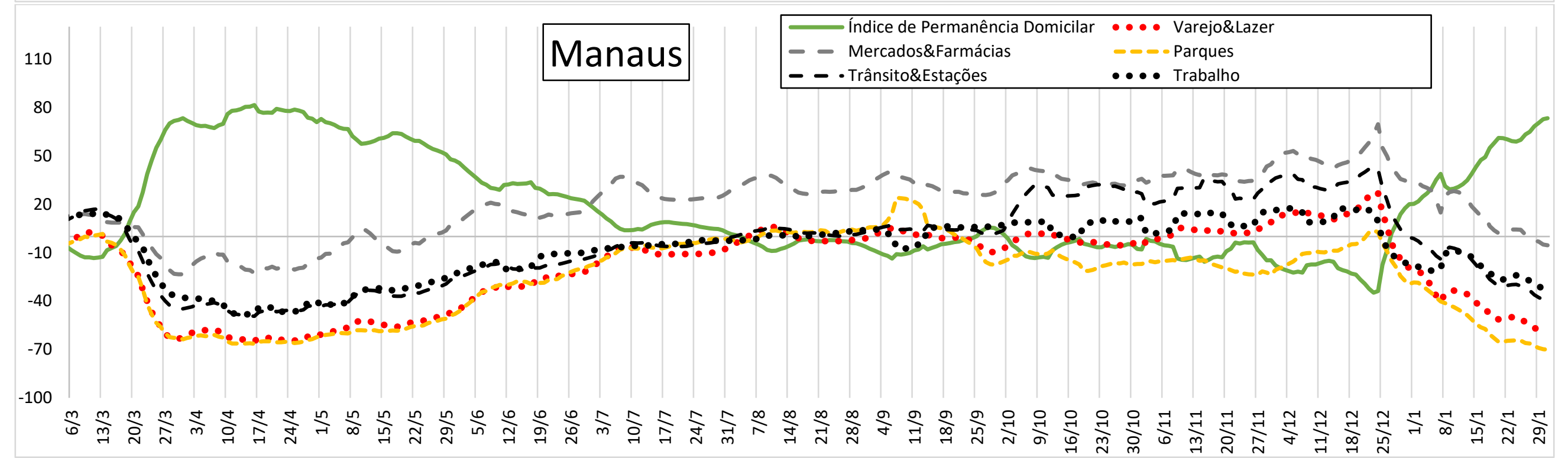

Figura 2 - Evolução do Índice de Permanência Domiciliar comparado com outros locais de permanência da população. Fortaleza e Manaus 2020-2021. 
No dia 02 de janeiro a Justiça do Amazonas acatou o pedido da Defensoria Pública e determinou a suspensão total das atividades consideradas não essenciais pelo prazo de 15 dias. O juiz previu até mesmo o uso de força policial para "preservar a ordem pública". No dia 14 de janeiro, o governador anunciou o Decreto $\mathrm{N}^{\circ}$ 23.282, que proíbe a circulação de pessoas em Manaus entre 19h e 6h. A medida entrou em vigor em 15 de janeiro e terá o prazo de 10 dias. No dia 27 de janeiro o Prefeito e a Secretária de Saúde de Manaus foram presos com base no gerenciamento inadequado da COVID-19 e da vacinação.

A sequência de fatos acima descritos, indica um desmando administrativo de tal ordem, que foi necessária a intervenção da Defensoria Pública e do Judiciário para restabelecer o isolamento social no dia 02 de janeiro de 2021, já na vigência do caos e do colapso do SUS no município. Quanto a permanência nas residências, o IPD, que apresentava valores negativos, evoluiu acentuadamente em apenas algumas semanas (Figura 1).

No entanto foi tarde demais: em dezembro de 2020 e janeiro de 2021, os sistemas de saúde público (SUS) e privado de Manaus entraram em colapso. Enquanto no período de abril até dezembro de 2020 (270 dias), foram notificadas 3.380 mortes por COVID-19 em residentes em Manaus, no mês de janeiro de 2021 (31 dias), foram notificados 2.195 óbitos, evidenciando novo e evitável pico acentuado da mortalidade por COVID-19 (Figura 3). Em 14 de janeiro, dezenas de pacientes morreram asfixiados devido à falta de oxigênio na rede pública hospitalar de Manaus, evento que chocou a população brasileira e toda a humanidade. O Ministério da Saúde e o governo do Estado do Amazonas acompanhavam a aceleração de casos, mas não tomaram medidas eficazes a tempo. Dias depois, essa tragédia se repetiu em outras cidades do interior do Amazonas.

Em Fortaleza as medidas legais de isolamento social vêm sendo prorrogadas por decretos estaduais desde agosto de 2020. O IPD é sempre superior ao registrado em Manaus, mas decai ao longo do mês de dezembro, atingindo valores muito baixos, reflexo de um aumento da permanência tanto em Parques como no Varejo\&Lazer no período das festas de fim de ano (Figura 2). Pelo Decreto de 09 de janeiro de 2021 ficam prorrogadas, no Estado do Ceará, as medidas de isolamento social até o dia 31 de janeiro de 2021. Logo após esse Decreto nota-se, também, um aumento correspondente do IPD, que subiu de modo consistente.

Comparativamente, no mês de janeiro ocorreram 181 óbitos em Fortaleza. A taxa de incidência de casos, que vinham aumentando a partir de 25 de dezembro, alcançou 25 por 100.000 habitantes dia 15 de janeiro, o mesmo nível alcançado em maio de 2020, logo antes dos elevados picos de incidência, e, sobretudo, de mortalidade, que ocorreram na cidade em junho de 2020 (Figura 1). Em Fortaleza, a permanência domiciliar foi consistentemente mais alta no período 
em estudo; baixou muito no período das festas de fim de ano, em decorrência de maior permanência em Mercados\&Farmácias, mas nunca atingiu valores abaixo de zero. Logo após o Decreto governamental de 08/01/2021, ampliando o isolamento até 31 de janeiro, nota-se um incremento no IPD em Fortaleza. Por razões que não puderam ser investigadas no presente estudo, a população de Fortaleza parece ter se convencido da importância das medidas de isolamento e aderiu às mesmas de modo consistente.

É importante destacar que, mesmo tendo mantido maiores Índices de Permanência Domiciliar, Fortaleza apresentou taxas de incidência em torno de 25 casos por 100.000 habitantes no mês de maio de 2020 e uma Taxa de Mortalidade de 3,5 por 100.000 habitantes, níveis considerados elevados (Figura 3).

\section{DISCUSSÃO}

O objetivo do presente trabalho foi analisar se as medidas legais dos governos locais e os níveis de isolamento social, podem ter contribuído para o colapso do SUS no município de Manaus, comparando com o comportamento da pandemia de COVID-19 em Fortaleza.

O Centro de Pesquisas e Estudos de Direito Sanitário (CEPEDISA) da Faculdade de Saúde Pública da Universidade de São Paulo publicou o "Boletim Direitos na pandemia: mapeamento e análise das normas jurídicas de resposta à COVID-19 no Brasil” no qual analisou as 3.049 normas publicadas no âmbito da União no ano de $2020^{16}$. Em grande parte, houve uma ênfase em medidas econômicas adotadas pelo governo federal nos meses de janeiro a junho de 2020 para enfrentamento da COVID-19. No referido período houve 317 produções de normas pelo ministério da economia, seguido pelos conselhos profissionais, com 308 publicações ${ }^{17}$. Poucas normas federais foram publicadas na área da saúde, segundo o CEPEDISA.

Nota-se que o município de Manaus não alcançou uma grande adesão ao isolamento social. Por outro lado, o estado do Ceará nos meses de março a maio de 2020 determinou medidas incisivas, como multa diária aos estabelecimentos não essenciais que abrissem, e a proibição de circulação de pessoas pela cidade, com objetivo de diminuir a incidência da doença. Por outro lado, nesse mesmo período, a $5^{\text {a }}$ Vara da Fazenda Pública determinou o funcionamento de salões de beleza no município de Manaus, considerando-os como serviço essencial ${ }^{\mathbf{1 6}}$. 


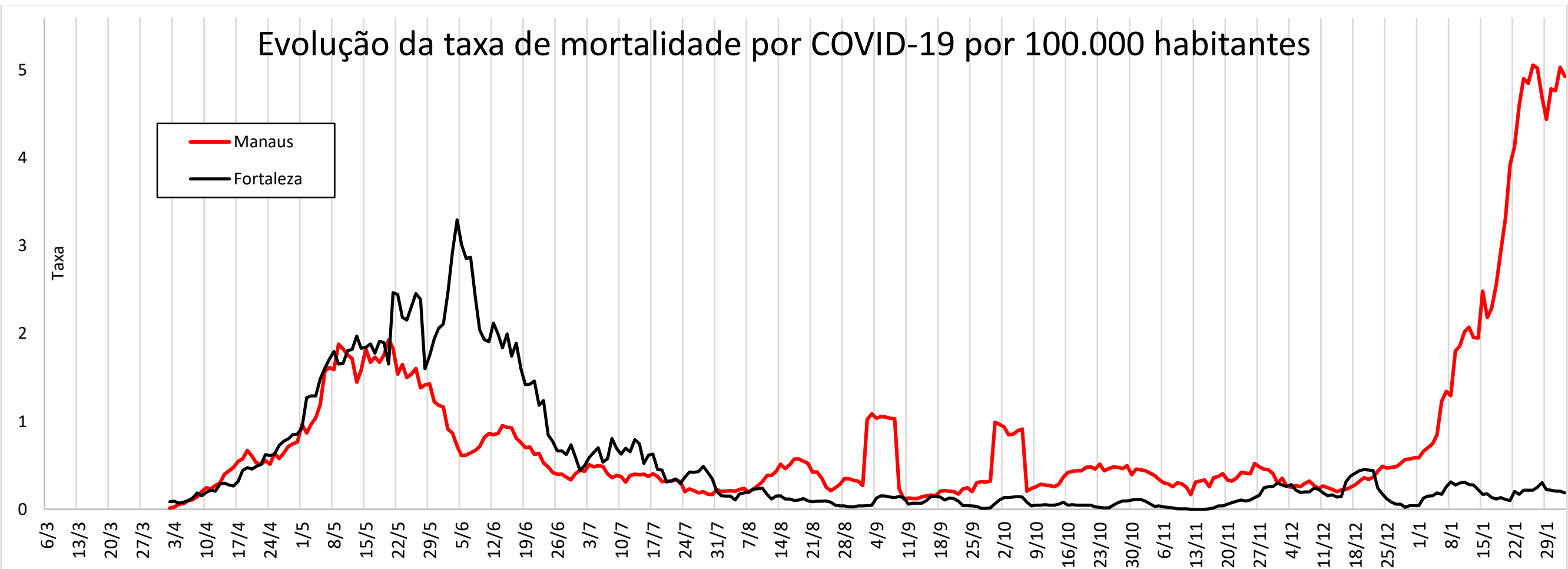

Figura 3 - Médias Móveis para os últimos 7 dias da taxa de mortalidade por COVID-19 por 100.000 habitantes. Fortaleza e Manaus 2020-2021. 
Um estudo de modelagem matemática realizado por $\mathrm{Li}$ et $\mathrm{al}^{18}$, que objetivou entender a associação da introdução e intensificação de medidas não farmacológicas com o nível de transmissão de SARS-CoV-2 em 131 países, medido pelo número de reprodução variável no tempo (R), evidenciou que medidas não farmacológicas isoladas, incluindo fechamento de escola, fechamento de local de trabalho, proibição de eventos públicos, proibição de reuniões de mais de dez pessoas, condições específicas para sair de casa e limites de movimento interno, estão associados à transmissão reduzida de SARS-CoV-2. Entretanto, os efeitos de a introdução e da intensificação destas medidas não farmacológicas só se manifestam depois de 1-3 semanas de sua implementação. Da mesma forma, o aumento na taxa de transmissão do vírus também sofre um atraso ao se suspender as medidas não farmacológicas, sendo este efeito ainda mais demorado. No presente estudo foi observado que a queda acentuada do IPD a partir de junho de 2020 em Manaus e Fortaleza, sendo particularmente acentuada no segundo município, coincidiu com aumento da taxa de incidência e do número absoluto de óbitos nos dois municípios a partir dos meses de novembro e dezembro. É preciso enfatizar que em nenhum momento houve interrupção da transmissão comunitária de COVID-19. Em janeiro de 2021 a taxa de incidência continua aumentando acentuadamente e a mortalidade assumiu dimensões catastróficas em Manaus.

Também, os reflexos políticos e a baixa aderência às medidas não farmacológicas para controle da COVID-19 no estado do Amazonas, desencadeou uma crise nos sistemas de saúde $\mathbf{2 1}$, que já apresentavam fragilidade, como no município de Manaus ${ }^{22}$. Além disso, o relaxamento das medidas de contenção durante o percurso da epidemia da COVID-19 tanto em Manaus como em Fortaleza pode ter sido um dos fatores para o aumento dos níveis epidêmicos nessas localidades $^{21}$. Tal fato pode ter contribuído para o surgimento de novas variantes no estado do Amazonas do Sars-CoV-2, com mutações na proteína Spike comum às linhagens B.1.1.7 e B.1.351, fato que tem gerado preocupações em todo o mundo, devido a uma probabilidade de maior transmissibilidade destas novas linhagens ${ }^{\mathbf{2 3}, 24}$.

Este estudo, de modo inédito, trouxe como contribuição a criação e análise do Índice de Permanência Residencial, a partir de dados de acesso livre. A relevância do IPD na interpretação da mobilidade populacional em dois grandes centros urbanos (epicentros da pandemia) foi demonstrada nesta pesquisa. A validade e sensibilidade deste novo indicador foi evidente, pois o mesmo responde, de modo rápido, às determinações legais de isolamento e de relaxamento. No entanto não estava no escopo do estudo analisar a relação do IPD com a aderência da população, nem aprofundar a análise de sua relação com a incidência e/ou número 
de óbitos, que dependem de vários outros fatores ligados à mídia (e Fake News) e à organização dos sistemas de saúde e de vigilância epidemiológica ${ }^{\mathbf{1 8}}$.

Outra consequência importante da Pandemia é o empobrecimento da população. As pessoas de classes econômicas mais baixas, que já viviam com salários parcos, durante a pandemia foram desligadas dos seus empregos ou tiveram seus salários reduzidos. Governos estaduais e municipais para tentar contornar essa situação isentaram a população de contas de água e energia elétrica, distribuíram cestas básicas e o governo federal por iniciativa dos parlamentares criou o auxílio emergencial, porém as condições de moradia e falta de saneamento não foram temas de discussão ou de ações nesse período ${ }^{19}$.

Entre os fatores limitantes deste estudo, cita-se o fato de não ter sido realizada uma coleta de dados de natureza qualitativa junto à população para investigar possíveis razões para aderência às medidas de isolamento nos municípios incluídos na pesquisa. Hipóteses plausíveis que não puderam ser investigadas são, por um lado, aspectos motivacionais e por outro até mesmo atitudes negacionistas quanto à pandemia. Outra limitação refere-se ao desafio de implantar medidas de distanciamento social, de controle sanitário (álcool gel, sabão para higiene das mãos) e de imposição de quarentena aos casos suspeitos e confirmados de COVID-19, em populações de muito baixa renda que habitam em aglomerações subnormais (favelas). Essas medidas pressupõem que o local de moradia é amplo, ventilado, tem água tratada e material de limpeza disponíveis, o que na maioria dos casos é uma falácia para a população de mais baixa renda ${ }^{19,20}$.

Apesar dos dados evidenciarem um maior percentual de aderência às medidas não farmacológicas em Fortaleza, quando comparada com o município de Manaus, um estudo realizado na região nordeste do Brasil relatou que na semana epidemiológica 24 de 2020 (7/06 a 13/06) a ocupação de leitos de UTI no estado do Ceará já chegava a $90 \%$. A partir de janeiro de 2021 ocorre aumento linear da incidência de COVID-19 em Fortaleza ${ }^{\mathbf{2 6}}$, e, em 9 de fevereiro de 2021, segundo dados do IntegraSUS Ceará, 91,8\% dos leitos de UTI na capital estão ocupados $^{27}$. Concluindo, impõe-se a aplicação rigorosa de medidas não farmacológicas, a realização de mais testes por habitantes, a realização de testagem em casos suspeitos de COVID-19, o rastreamento de contatos de casos confirmados de COVID-19, a realização de quarentena por pessoas confirmadas ou sob suspeita de COVID-19, o aumento da vigilância genômica do vírus no Brasil, além da imunização massiva da população brasileira. Sem estas medidas, infelizmente, ainda haverá milhares de mortes a lamentar no futuro próximo. 
CONTRIBUIÇOES DOS AUTORES: BARRETO ICHC participou da pesquisa, contribuiu na metodologia, na escrita do rascunho e na revisão do texto final; COSTA FILHO RV participou da pesquisa, contribuiu na metodologia, na escrita do rascunho e na revisão do texto final; RAMOS RF contribuiu na metodologia, na interpretação dos resultados e na revisão final do texto; OLIVEIRA LG contribuiu na pesquisa, na discussão dos resultados e na revisão final; MARTINS NRV contribuiu na pesquisa, na discussão dos resultados e na revisão final; CAVALCANTE FV contribuiu na pesquisa, na escrita do artigo e na revisão final; ANDRADE LOM contribuiu na escrita do artigo e na revisão final; SANTOS LMP concebeu a pesquisa, contribuiu na metodologia, na interpretação dos resultados, na escrita do rascunho e na revisão do texto final.

AGRADECIMENTOS: Agradecemos aos Ministérios da Ciência e Tecnologia e Ministério da Saúde, que por meio da Chamada Pública MCTI/CNPq/CT-Saúde/MS/SCTIE/Decit N ${ }^{\circ}$ 07/2020 financiou o presente trabalho. Agradecemos também aos colegas da UNB: Mauro Sanchez; Ivan Zimmermann e Everton Nunes; da ENSP/FIOCRUZ: Claudia Pereira; da Fiocruz/AM: Fernando Herkrat e Rodrigo Lima, pelas contribuições na discussão dos resultados iniciais do estudo.

CONFLITOS DE INTERESSE: Os autores declaram a ausência de conflitos de interesse.

\section{REFERÊNCIAS BIBLIOGRÁFICAS:}

1. Brasil. Ministério da Saúde. Coronavírus COVID-19: Diretrizes para diagnóstico e tratamento da COVID-19 [Internet]. 2 versão. SCTIE, editor. Brasília: Ministério da Saúde; 2020. 73 p. Available from: https://saude.rs.gov.br/upload/arquivos/202004/14140600-2-msdiretrizes-covid-v2-9-4.pdf

2. WHO. Statement on the second meeting of the International Health Regulations (2005) Emergency Committee regarding the outbreak of novel coronavirus (2019-nCoV) [Internet]. 2020. Available from: https://www.who.int/news/item/30-01-2020-statement-on-the-secondmeeting-of-the-international-health-regulations-(2005)-emergency-committee-regarding-theoutbreak-of-novel-coronavirus-(2019-ncov)

3. CEPEDES/ENSP/FIOCRUZ. A gestão de riscos e governança na pandemia por COVID-19 no Brasil: análise dos decretos estaduais no primeiro mês, relatório técnico e sumário executivo [Internet]. Rio de Janeiro: Fiocruz; 2020. Available from: https://www.arca.fiocruz. br/bitstream/icict/41452/2/relatorio_cepedes_gestao_riscos_covid19_final.pdf

4. Garcia PJ, Alarcón A, Bayer A, Buss P, Guerra G, Ribeiro H, et al. COVID-19 Response in Latin America. Am J Trop Med Hyg [Internet]. 2020 Nov 4;103(5):1765-72. 
5. Brasil. COVID19: Painel Coronavírus [Internet]. Ministério da Saúde. 2021 [Access 2021 Jan 25]. Available from: https://covid.saude.gov.br/

6. Goya N, Andrade LOM de. O Sistema Único de Saúde e o desafio da gestão regionalizada e contratualizada. Rev Bras em Promoção da Saúde [Internet]. 2018 Dec 21;31(4).

7. CONASS. Nota à imprensa - Aquisição de vacinas contra a Covid-19 por empresas [Internet]. 2021. Available from: https://www.conass.org.br/nota-a-imprensa-aquisicao-devacinas-contra-a-covid-19-por-empresas/

8. Brasil. Ranking IDHM Municípios 2010 [Internet]. PNUD. 2010 [Access 2021 Jan 27]. Available from: https://www.br.undp.org/content/brazil/pt/home/idh0/rankings/idhmmunicipios-2010.html

9. IBGE. Panorama Cidades [Internet]. IBGE. 2020 [Access 2021 Jan 27]. Available from: https://cidades.ibge.gov.br/brasil/panorama

10. IBGE. Estimativa da População [Internet]. IBGE. 2020 [Access 2021 Jan 27]. Available from: https://www.ibge.gov.br/estatisticas/sociais/populacao/9103-estimativas-depopulacao.html?=\&t=resultados

11. Brasil. CNESNet [Internet]. Datasus. 2021 [Access 2021 Jan 27]. Available from: http://cnes2.datasus.gov.br/

12. IBGE. Censo Demográfico [Internet]. 2010. Available from: https://www.ibge.gov.br/ estatisticas/sociais/populacao/9662-censo-demografico-2010.html?edicao=9754\&t=resultados

13. Google. COVID-19: Relatórios de mobilidade da comunidade [Internet]. Google. 2020 [Access 2021 Jan 29]. Available from: https://www.google.com/covid19/mobility/

14. IBGE/SIDRA. Estimativas de População: Tabela 6579 - População residente estimada [Internet]. IBGE. 2020 [Access 2020 Jan 20]. Available from:

https://sidra.ibge.gov.br/tabela/6579

15. LeisMunicipais. Tenha conhecimento das Leis criadas pelo seu município e Governo do Estado, a respeito do novo Coronavírus (COVID-19) [Internet]. LeisMunicipais. 2020 [Access 2021 Jan 20]. Available from: https://leismunicipais.com.br/coronavirus

16. Cepedisa \& Conectas. Direitos na Pandemia: Mapeamento e Análise das Normas Jurídicas de Resposta à COVID-19 no Brasil [Internet]. Boletim no 10. Asano CL, Ventura D de FL, Aith FMA, Reis RR, Ribeiro TB, editors. São Paulo: CEPEDISA; 2021. 42 p. Available from: http://cepedisa.org.br/wp-content/uploads/2020/07/02boletimcovid_PT_06.pdf

17. Cepedisa \& Conectas. Direitos na Pandemia: Mapeamento e Análise das Normas Jurídicas de Resposta à COVID-19 no Brasil [Internet]. Boletim n ${ }^{\circ}$ 2. Asano CL, Ventura D de FL, Aith FMA, Reis RR, Ribeiro TB, editors. São Paulo: CEPEDISA; 2020. Available from: http://cepedisa.org.br/wp-content/uploads/2020/07/02boletimcovid_PT_06.pdf 
18. Li Y, Campbell H, Kulkarni D, Harpur A, Nundy M, Wang X, et al. The temporal association of introducing and lifting non-pharmaceutical interventions with the time-varying reproduction number $(\mathrm{R})$ of SARS-CoV-2: a modelling study across 131 countries. Lancet Infect Dis [Internet]. $2021 \mathrm{Feb}$;21(2):193-202. Available from:

https://linkinghub.elsevier.com/retrieve/pii/S1473309920307854

19. Calmon TVL. As condições objetivas para o enfrentamento ao COVID-19: abismo social brasileiro, o racismo, e as perspectivas de desenvolvimento social como determinantes. NAU Soc [Internet]. 2020 Apr 30;11(20):131.

20. Patel JA, Nielsen FBH, Badiani AA, Assi S, Unadkat VA, Patel B, et al. Poverty, inequality and COVID-19: the forgotten vulnerable. Public Health [Internet]. 2020 Jun;183:110-1.

21. Albuquerque B, Freitas CM de, Barcellos C, Villela DAM, Naveca FG, Herkrath F, et al. Nota Técnica no 04. Reflexões sobre o comportamento da epidemia da COVID-19 segundo as regiões de saúde do Estado do Amazonas [Internet]. COVID-19 I, editor. Rio de Janeiro: Fiocruz; 2021. Available from: https://www.arca.fiocruz.br/handle/icict/45840

22. Galvao TF, Tiguman GMB, Caicedo Roa M, Silva MT. Inequity in utilizing health services in the Brazilian Amazon: A population-based survey, 2015. Int J Health Plann Manage [Internet]. 2019 Oct 12;34(4).

23. Fiocruz. Nota Técnica 2021/01 - Rede Genômica Fiocruz / Ministério da Saúde [Internet]. Ministério da Saúde, editor. Rio de Janeiro: Fiocruz; 2021. Available from: https://portal.fiocruz.br/sites/portal.fiocruz.br/files/documentos/nota_tecnica_ms_2021-01-12.pdf

24. Sabino EC, Buss LF, Carvalho MPS, Prete CA, Crispim MAE, Fraiji NA, et al. Resurgence of COVID-19 in Manaus, Brazil, despite high seroprevalence. Lancet [Internet]. 2021 Feb;397(10273):452-5. Available from: https://linkinghub.elsevier.com/retrieve/pii/S0140673621001835

25. Ximenes RA., Albuquerque MFP., Martelli CMT, Thália VB de A, Filho DB., Souza WV, et al. COVID-19 no Nordeste do Brasil: entre o lockdown e o relaxamento das medidas de distanciamento social. Cien Saude Colet [periódico na internet] [Internet]. 2021;Jan. Available from: http://www.cienciaesaudecoletiva.com.br/artigos/covid19-no-nordeste-dobrasil-entre-o-lockdown-e-o-relaxamento-das-medidas-de-distanciamentosocial $/ 17913$ ? $\mathrm{id}=17913 \& \mathrm{id}=17913 \& \mathrm{id}=17913$

26. Fortaleza. Prefeitura Municipal. Secretaria Municipal de Saúde. Coordenadoria de Vigilância à Saúde. Boletim Epidemiológico. Inf Sem COVID-19 [Internet]. 2021,33 semana epid. Available from: https://coronavirus.fortaleza.ce.gov.br/boletim-epidemiologico.html 27. Ceará. Histórico de Internações por COVID-19 [Internet]. IntegraSUS. 2021. Available from: https://indicadores.integrasus.saude.ce.gov.br/indicadores/indicadorescoronavirus/historico-internacoes-covid. 